\title{
Ovarian thecoma- an incidental finding in a patient with UVprolapse
}

\author{
Suniti Rawal, Kesang D Bista, Geeta Gurung, Ashma Rana \\ Dept of Obs/Gyn, TU Teaching Hosp, Kathmandu. Nepal
}

\begin{abstract}
:
A seventy two year old postmenopausal multiparous lady who presented with $11 \mathrm{I}^{\circ}$ uterovaginal prolapse was found to have a solid mass palpable through the vaginal fornices for which the vaginal procedure was deferred for abdominal hysterectomy. A solid mass resembling fibrothecoma was discovered which after histopathological examination was reported to be thecoma pointing out the importance of preoperative evaluation.
\end{abstract}

Keywords: post menopausal ovarian tumor, thecoma, uterovaginal prolapse with ovarian tumor

\section{Introduction}

Thecomas or theca cell tumors of the ovary are reported in a very small number of women constituting about $2 \%$ of all ovarian tumors. These are benign stromal neoplasms and are well circumscribed, solid, unilateral tumors usually occurring in the $5^{\text {th }}$ to $6^{\text {th }}$ decade of life (early postmenopausal years). ${ }^{1}$ Not every case of stromal tumors is associated with abnormal uterine bleeding although hormone production (estrogen) can lead to endometrial hyperplasia and adenocarcinoma of endometrium. This however, is an example where the patient presented with discomfort caused by uterovaginal prolapse.

\section{Case report}

A 72 year old multiparous lady was admitted with the diagnosis of III $^{\circ}$ uterovaginal prolapse with cystocele, enterocele and rectocele to undergo vaginal hysterectomy with pelvic floor repair. She was menopausal for the past 25 years. Her abdomen was soft and per vaginal examination showed the uterus was atrophic, retroverted, firm, nontender and mobile. A hard mass measuring about $5 \times 4 \mathrm{~cm}$ was palpable through the right fornix in the right adenexa. Transvaginal sonography (TVS) revealed a complex solid mass in the right adenexa with solid components and without ascites during the preoperative investigations. So in the place of vaginal hysterectomy with pelvic floor repair, a total abdominal hysterectomy (TAH BSO) with bilateral salpingooophorectomy was performed because of the ovarian tumor.
The per operative findings were consistent with atrophic uterus of $4 \times 4 \mathrm{~cm}$, with few areas of hemorrhage. The right ovary was enlarged to $5 \times 4.5 \mathrm{~cm}$ in size, solid, white, hard and smooth with prominent vessels (fig 1). The left ovary and both the tubes were normal. The omentum, liver and the under surface of the diaphragm were also normal.

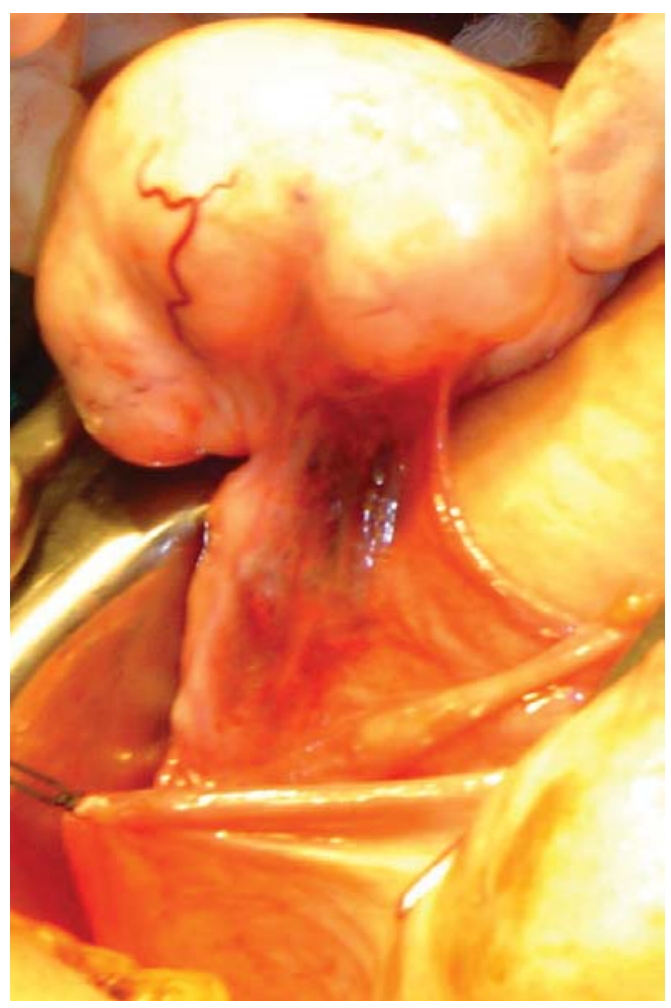

Figure 1 Thecoma of ovary 
The cut section of the tumor was hard and pearly white (Fig 2). During the post operative period she developed burst abdomen which was re-sutured and she was discharged after removal of the suture. The histological examination of the specimen showed right ovarian mass with solid growth of stroma (spindle shaped cells), blunt ended nuclei, ill-defined cytoplasm with mitotic figures 2 per 10 high power field consistent with ovarian thecoma. The left ovary was found to be cystic.

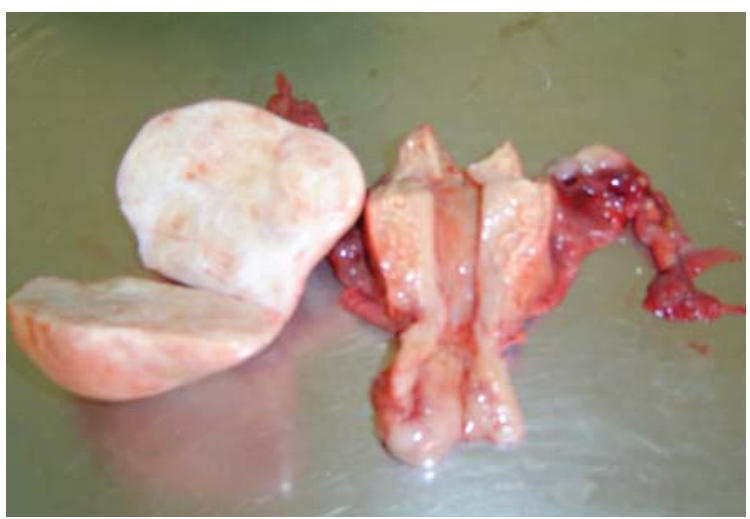

Figure 2 Cut section of thecoma

\section{Discussion}

The diagnosis of thecoma was not considered initially in the preoperative period due to its low incidence in our hospital and this being the first case deserved to be reported. ${ }^{2}$

These are essentially benign tumor with low malignant potentials. Yet very rarely malignant thecomas have been reported as well, which have a poor prognosis and require systemic chemotherapy or radiotherapy to improve their survival. Wu L et al in China (1958-98), collected a total of 74 cases of ovarian thecomas, out of which 8 cases had malignant thecoma the rest being benign. The mean age of incidence was 52 years and most of them had presented with abdominopelvic mass, metromenorrhagia, abnormal vaginal bleeding, pain abdomen and infertility. Twenty three of them had ascites, 1 each with hydrothorax, endometrial adenocarcinoma, endometrial dysplasia and rest with myoma, polyps and endometrial hyperplasia. They received treatment accordingly like surgery, chemotherapy and radiation. ${ }^{1}$

Benign ovarian thecoma associated with a large quantity of ascites, about 9L of straw colored ascitic fluid, (atypical Meig's syndrome) and elevated serum CA 125 and CA 15-3 has also been reported. ${ }^{3}$

This patient did not have ascites and assuming the tumor to be a benign one, we did not opt for the tumor markers. Our patient was lucky enough not to have any of these symptoms, signs and the associated diseases; and the prolapse was actually a boon in disguise that lead to the diagnosis of this thecoma, that was treated completely with surgery.

\section{Conclusions}

This case points out the importance of pre-operative evaluation as sometimes a problematic but rare lesion is overlooked in the presence of a more common problem, which in this case was uterovaginal prolapse.

\section{References}

1. Wu L, Zhang W, Li H, Li L, Kong W, Liu L. Clinical analysis of 74 cases with ovarian thecoma. Zhonghua Fu Chan Ke Za Zhi. 2002 Feb; 37(2):101-3.

2. Jha R, Shrestha B, Singh M. Ovarian Granulosa cell tumor. Journal of Society of Surgeons of Nepal 2001; 4: 77-9.

3. Renaud MC, Plante M, Roy M. Ovarian thecoma associated with large quantity of ascites and elevated serum CA 125 and CA15-3. J Obstet Gynaecol Can. 2002 Dec; 24(12):963-5. 